\title{
Sentir de alumnos universitarios y bachillerato luego de 17 meses de confinamiento por covid-19
}

Irene Zapata Silva

irene.zs@slp.tecnm.mx

María Guadalupe Hernández Sierra

maria.hs@slp.tecnm.mx

Blanca Estela Ramírez Gámez

blanca.rg@slp.tecnm.mx

Elizabeth Rivera Bravo elizabeth.rb@slp.tecnm.mx

Myriam Araceli Martínez Rodríguez

miriam.mr@slp.tecnm.mx

Tecnológico Nacional de México

campus Instituto Tecnológico de San Luis Potosí

Soledad de Graciano Sánchez, S.L.P., México

Raúl Guillermo Coronel Delgadillo raul.coronel@cecyteslp.edu.mx

Colegio de Estudios Científicos y Tecnológicos del Estado de San Luis Potosí San Luis Potosí, S.L.P., México

Estudiante: Luis Martín Rodríguez Vega

L17180359@slp.tecnm.mx Tecnológico Nacional de México campus Instituto Tecnológico de San Luis Potosí

Soledad de Graciano Sánchez, S.L.P., México

\section{RESUMEN}

El objetivo del trabajo fue conocer el sentir de los alumnos universitarios y bachillerato luego de 17 meses de confinamiento por COVID-19. Es de tipo cuantitativo, se aplicó una encuesta en línea a 648 discentes utilizando estadística descriptiva para la recolección de datos y su posterior análisis. Los resultados muestran que la mayoría manifiesta no ver afectado su estado de salud. 287 se sienten estresados o tensos y 230 aburridos o desinteresados. Sin embargo 265 valoran a su familia y 222 la vida. La comunidad estudiantil reconoce no haber tenido afectaciones en el núcleo familiar (334) aunque, 96 
dicen tener mala comunicación y 143 tener disgustos y conflictos por cambios de hábitos de sueño. 278 de los encuestados están temerosos de contagiarse y contagiar a sus familiares, contrariamente 243 están entusiasmados por la reanudación de actividades escolares. 180 se manifiestan ansiosos y 169 inseguros por la expectativa de retorno. El compromiso de cuidar la salud es de todos. Considerando la etapa de adultez emergente de los encuestados, su grado de compromiso es mayor que el de los niveles de estudio precedentes, lo que representa una ventaja para garantizar el regreso seguro a la educación presencial.

Palabras clave: adultez emergente; socialización presencial; covid 19; contacto físico; salud emocional 


\title{
Feelings of university and high school students after 17 months of confinement by covid-19
}

\begin{abstract}
The objective was to know about feelings of university students after 17 months of confinement for COVID-19. An online survey was applied to 648 students using descriptive statistics for data collection and subsequent analysis.

The results showed that health condition was not affected. 287 feel stressed or tense and 230 feel bored or disinterested. However, 265 value their family and 222 value their life. The student community acknowledges not having had any affectations in the family nucleus (334), although, 96 say they have poor communication and 143 have dislikes and conflicts due to changes in sleeping habits. 278 of those surveyed are fearful of infect themselves and their families, on the contrary 243 are enthusiastic about the resumption of school activities. 180 are anxious and 169 insecure due to the expectation of return. The commitment to take care of health belongs to everyone. Considering the emerging adulthood stage of the respondents, their commitment is greater than previous levels of study, which represents an advantage to guarantee the safe return to face-to-face education.
\end{abstract}

Keywords: emerging adulthood; face-to-face socialization; covid 19; physical contact; health

Artículo recibido: 02 Setiembre. 2021 Aceptado para publicación: 30 Setiembre. 2021 Correspondencia: irene.zs@ slp.tecnm.mx Conflictos de Interés: Ninguna que declarar 


\section{INTRODUCCIÓN}

En 2020, 1.500 millones de estudiantes en 188 países / economías fueron excluidos de sus escuelas. Los estudiantes de todo el mundo se han enfrentado a escuelas que están abiertas un día y cerradas al siguiente, causando una interrupción masiva de su aprendizaje. Con la pandemia de coronavirus (COVID-19) aún en su apogeo, la interrupción de la educación se ha extendido hasta 2021 y muchos sistemas educativos todavía están luchando para garantizar la continuidad del aprendizaje (OECD, 2021).

Los jóvenes que se encuentran en la etapa de adultez emergente, inscritos en nivel medio superior y superior, enfrentan desafíos propios de la transición a la edad adulta. Este ciclo se acompaña de cambios físicos y mentales en esta etapa existe alto riesgo de desarrollar problemas de salud mental ya que según menciona (Pariente, 2021) el 75\% de los trastornos mentales se desencadena entre los 18 y 29 años. Esta fase de la vida puede implicar algunos cambios como la migración de la localidad de origen, el distanciamiento familiar, la incursión en empleo de medio tiempo y sobre todo la incertidumbre en el futuro inmediato. El estrés se hace presente debido principalmente a las actividades que demanda la escuela: carga académica, largas horas de estudio y noches sin dormir. Se puede leer en (Pediatrics, 2021): "Los adultos jóvenes pueden tratar de esconder sus problemas por miedo, vergüenza o sentirse responsables de evitar ser una carga para otros."

El conocimiento de este escenario presenta para docentes, tutores, padres de familia y autoridades educativas una oportunidad para la prevención, detección y tratamiento oportuno de los trastornos mentales que garanticen el bienestar del mañana y el rendimiento académico actual y ocupacional a futuro. Al respecto se ignoran las consecuencias y afectaciones psicológicas relevantes en los estudiantes.

Además de la crisis sanitaria provocada por la COVID-19 surge una crisis de salud mental. Es urgente ocuparse de esos dos elementos. Las dificultades emocionales que sufren los adolescentes se ven afectadas por el estrés propio del confinamiento, por el aburrimiento y el desinterés, por las características modificadas en el ámbito del hogar, por la valoración que otorgan a la vida y a la familia, el aislamiento social, la interrupción de la educación presencial y la inseguridad e incertidumbre sobre los tiempos venideros. Los motivos al respecto tienen relación con desconocer qué pasará con la economía del país, con la situación económica familiar, ignorar qué ocurre y qué pasará en un futuro incierto, la 
inapropiada información noticiosa y los rumores que en medios y redes sociales circulan en estos días (Fernández, 2020).

Esto ocurre en momentos críticos de su desarrollo emocional y, en algunos casos, se suma a un aumento de los abusos que padecen (Jiménez, 2019). "Los universitarios se encuentran en un periodo en el que es mucho más relevante el contacto físico y la socialización presencial", explica (Mac-Ginty, 2021) y continua, "Las condiciones de encierro para muchos ha sido de hacinamiento y se han provocado tensiones en los grupos familiares". Los resultados, recopilados en un estudio publicado en la revista de psiquiatría Sopnia, revelaron que tres de cada cuatro estudiantes $(77,7 \%)$ reportaba que su estado de ánimo estaba peor o mucho peor en comparación al contexto pre pandémico (Mac-Ginty, 2021). A su vez en la revista 2020 se menciona que "un 63\% de los estudiantes indicó sentir aburrimiento, un $41 \%$ manifestó sentir ansiedad o estrés, seguido por frustración y molestia (35\%); mientras que un $21 \%$ dijo sentir tranquilidad y sólo un 3\% indicó sentirse "feliz de estar en casa" (Educación 2020, 2020).

Al inicio de la pandemia la decisión acerca de la clausura temporal de las instituciones educativas se decretó por el principio de salvaguardar la salud pública para evitar grandes acumulaciones de personas. Durante estos primeros meses fue agradable para los estudiantes, pero a 17 meses de confinamiento manifiestan diferente sentir (UNESCO, 2020).

\section{ESTRATEGIAS METODOLÓGICAS O MATERIALES Y MÉTODOS}

Según establece (Hernández-Sampieri R., 2018) para la identificación de los métodos de investigación; el estudio es de tipo cuantitativo, ya que se aplicó una encuesta en línea para conocer el sentir de los estudiantes tras 17 meses de confinamiento y sin la reapertura aun de las instituciones educativas de nivel medio superior y superior. Se utilizó la estadística descriptiva para la recolección de datos y posterior análisis de estos. El trabajo realizado es un acercamiento al aspecto de las emociones estudiantiles durante el aislamiento.

\section{Método y organización del trabajo de campo}

Por las características del grupo de estudio, se decidió llevar a cabo el siguiente plan de acción:

a) Elaboración del cuestionario con la herramienta Microsoft Forms de Google, de manera que fuera posible su difusión ágil y segura. La encuesta se diseñó con tres tipos de respuestas: 
- De solo una opción

- Escala de Likert

- De varias opciones

b) Distribución por medios electrónicos del enlace para responder la encuesta. Se envió a 15 instituciones educativas de nivel superior y a 7 de nivel medio superior, localizadas en los estados de: Chiapas, Chihuahua, Ciudad de México, Durango, Michoacán, Oaxaca, Puebla, Quintana Roo, San Luis Potosí, Sonora, Tamaulipas y Veracruz. Se obtuvieron 648 respuestas, de las cuales 462 corresponden a estudiantes de universidad y 186 de bachillerato.

c) Recopilación y análisis de los datos.

\section{RESULTADOS Y DISCUSIÓN}

Durante la primera infancia la atención de padres y cuidadores es indispensable. Al ser humano no le es posible sobrevivir sin comida, sueño y afecto. Como el primer sentido que se desarrolla es el tacto, y este se percibe a través de la piel, la cual está íntimamente ligada al sistema nervioso, es importante para el bebé sentir el contacto físico desde el momento de su nacimiento en forma de abrazos y caricias. El contacto piel con piel incide en su bienestar y es una valiosa fuente de salud mental para el resto de su vida (Torrecilla, 2020). Según el artículo publicado en el sitio Fundación Baltazar y Nicolás (Fundación Baltazar y Nicolás. Contribuyendo al desarrollo infantil temprano, 2020), "Si un bebé no recibe afecto, carece de estímulos para exteriorizar sus energías, pierde interés por mantener contacto visual, alimentarse o comunicar, sentirá que el mundo es hostil, y esa sensación perdurará hasta la adultez, complicándosele emocionalmente la vida. El adulto independiente primero fue un bebé dependiente".

En la etapa crucial en que se encuentran los jóvenes de 18 a 29 años es necesaria la sociabilización presencial con sus pares. Al ser seres sociales, solo se está completo al tener contacto físico con los demás ya que éste genera una serie de beneficios emocionales mejorando la salud. La situación actual exige el distanciamiento entre personas, está prohibido saludar con un abrazo o de mano, besar a quienes se quiere, recibir masaje y otros gestos parecidos; de ahí que los jóvenes estén deseosos de volver a la normalidad para tener nuevamente esa oportunidad de sentirse.

La distancia social, impuesta por las circunstancias y la cuarentena, no es beneficiosa. Incluso a nivel fisiológico se ha comprobado que el contacto físico aumenta la 
producción de serotonina, dopamina y oxitocina. Las tres son hormonas y están asociadas al bienestar físico y emocional (Torrecilla, 2020).

En la investigación realizada, los resultados obtenidos muestran que, del 100 por ciento de las respuestas obtenidas, 186 que, corresponden al 29 por ciento son estudiantes del nivel medio superior y 462 al 71 por ciento del nivel superior.

Figura 1. Nivel de estudios

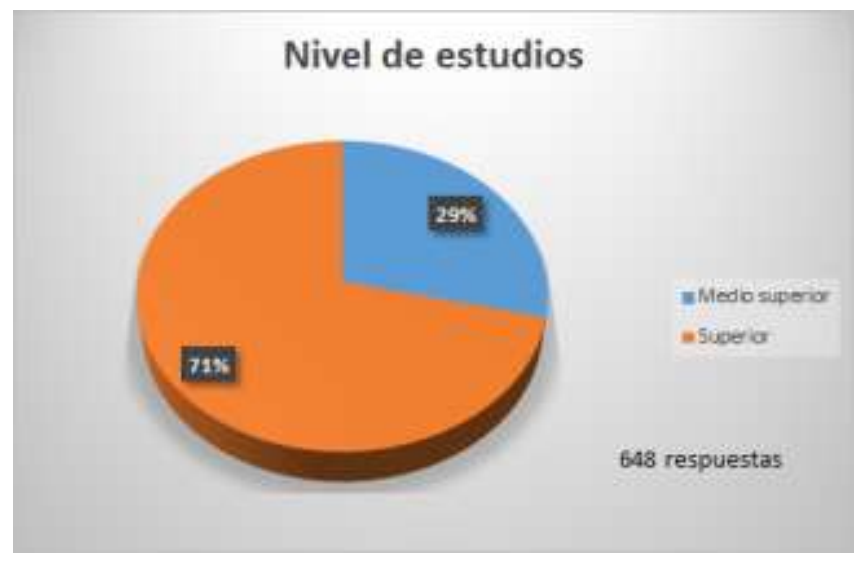

Fuente: Elaboración propia

A la pregunta: ¿En qué grado manifiestas que el confinamiento ha afectado tu estado de salud?

Las posibles respuestas están dadas en escala de Likert, donde: 1es nada afectado, 2 poco afectado, 3 neutral, 4 muy afectado y 5 totalmente afectado. Se puede observar que la mayoría de los alumnos (190) manifiesta que no se ha afectado su estado de salud y otro número importante considera que se ha afectado nada (180) o poco (147), según se aprecia en la tabla 1 y figura 2 .

\section{Tabla No. 1. Grado de afectación del estado de salud por el confinamiento}

\begin{tabular}{|c|c|}
\hline Nivel de afectación & Número de alumnos \\
\hline 1 & 180 \\
\hline 2 & 147 \\
\hline 3 & 190 \\
\hline 4 & 93 \\
\hline 5 & 38 \\
\hline Total & $\mathbf{6 4 8}$ \\
\hline
\end{tabular}

Figura 2. Grado de afectación 


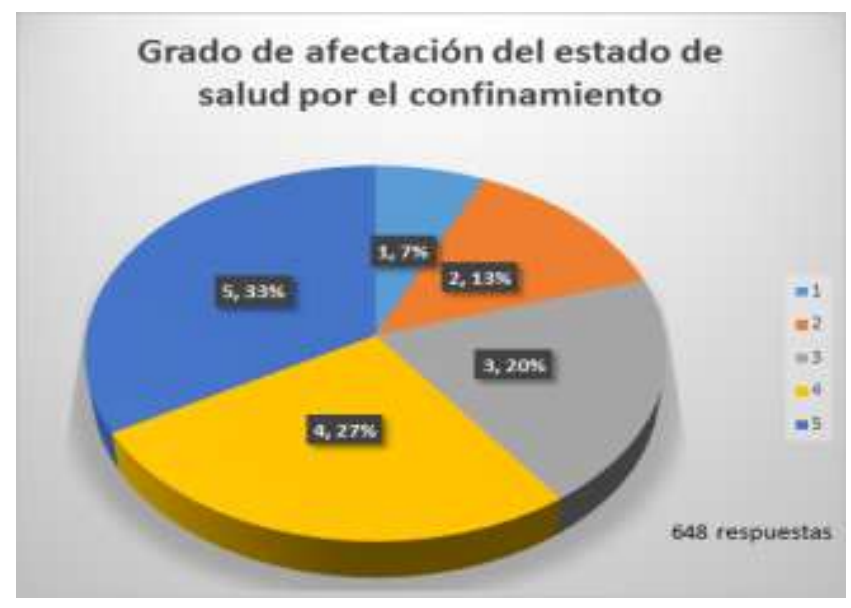

Fuente: Elaboración propia

En relación con los adjetivos o enunciados que definen a los encuestados, se puede observar que los indicadores más altos corresponden a 287 alumnos que se sienten estresados o tensos y 230 aburridos o desinteresados. Sin embargo 265 valoran a su familia y 222 la vida, siendo estos aspectos positivos según se muestra en la figura 3.

Figura 3. Adjetivos y enunciados que definen a los estudiantes

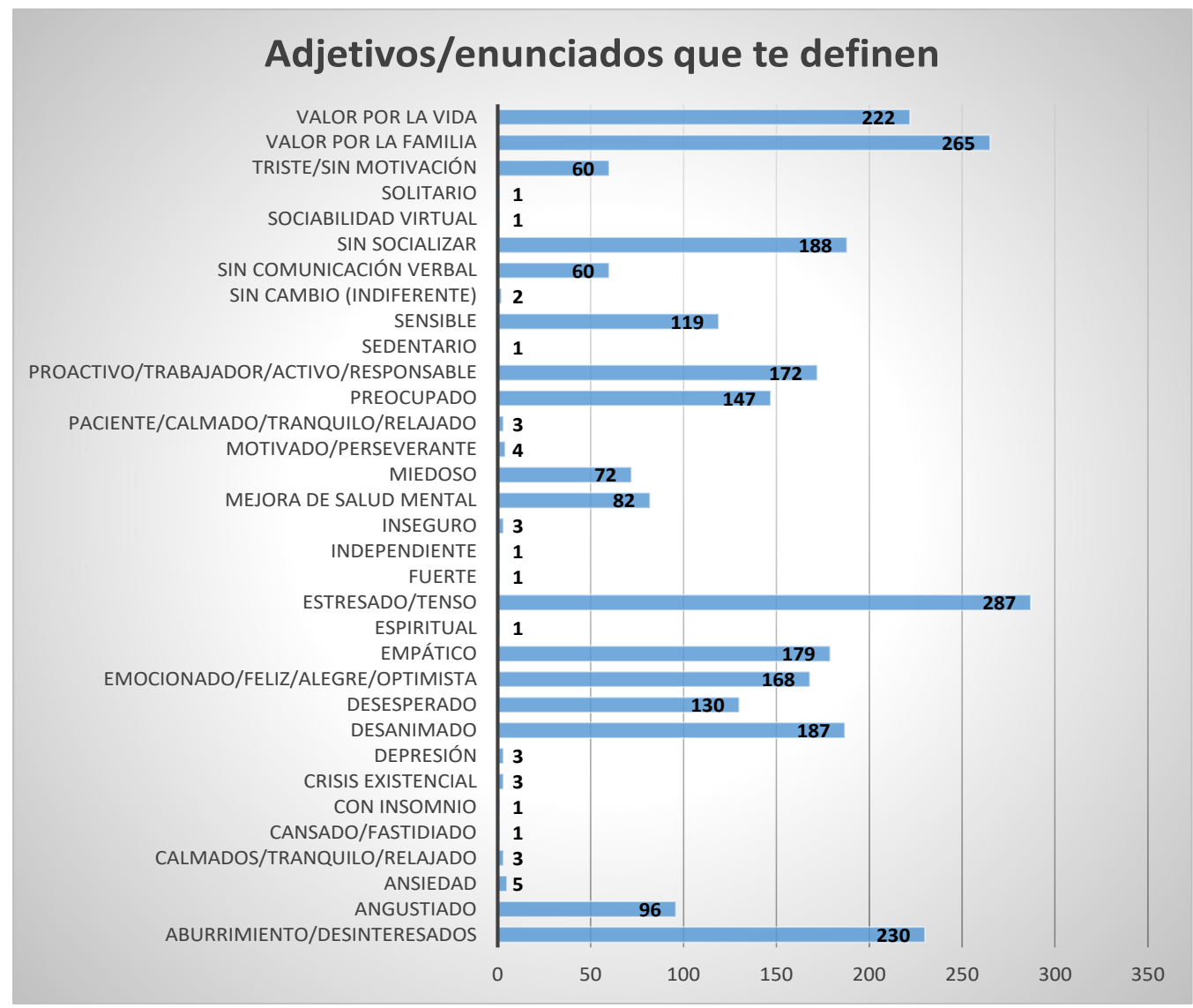

Fuente: Elaboración propia 
El síntoma más presente es la ansiedad seguido de la preocupación, desesperación, desánimo generalizado, depresión, tristeza, molestias físicas y deseo de comer en exceso. Aun así 145 estudiantes no presentan síntomas, según se observa en la figura 4.

Figura 4. Síntomas que presentan los estudiantes

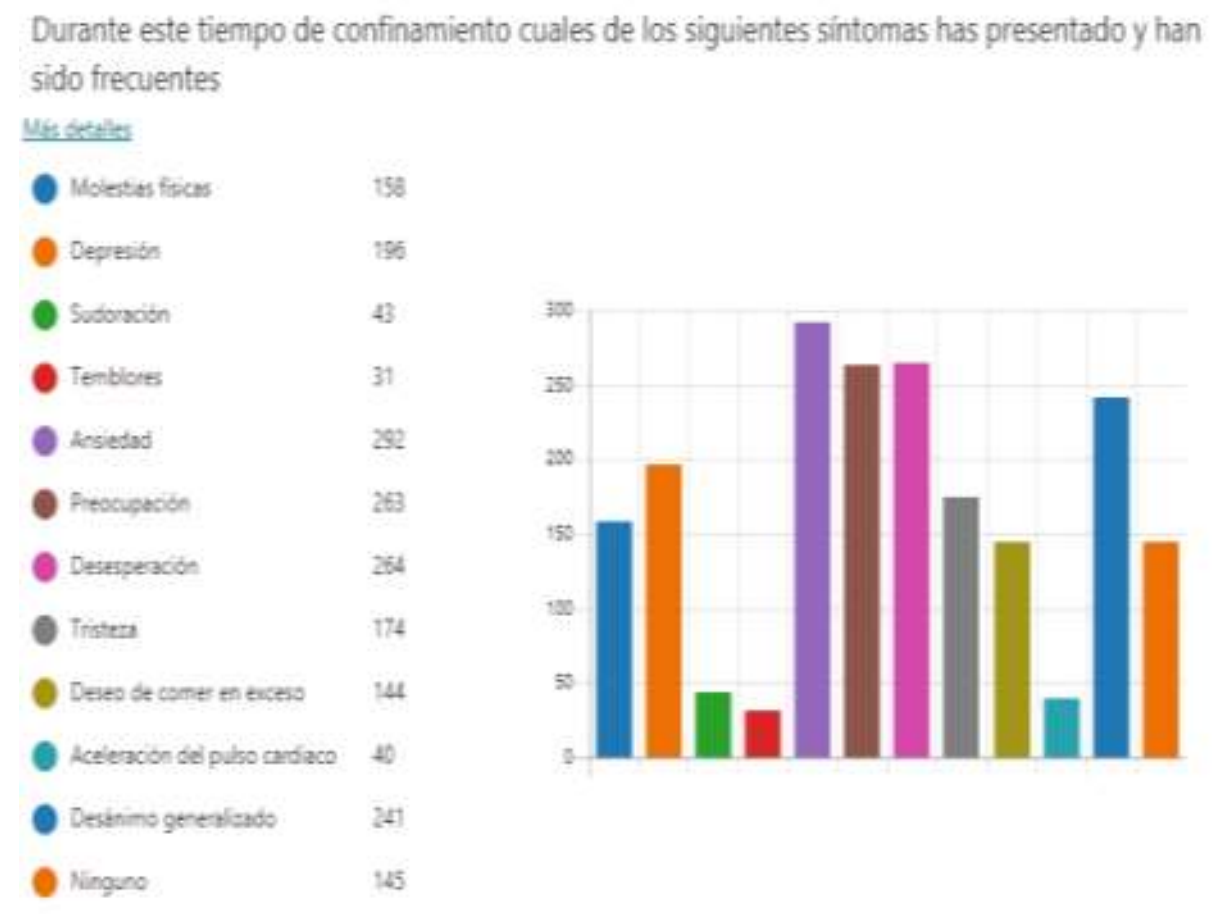

Fuente: Elaboración propia

Al prohibirse las reuniones debieron abandonarse las actividades extraescolares, siendo éstas parte importante del esparcimiento de los jóvenes y contribuían a menguar el estrés y fomentan el sentido de pertinencia. La tabla número 2 muestra este grado de afectación. Se utilizó la escala de Likert para que los encuestados manifestaran su grado de afectación. Dónde: les nada afectado, 2 poco afectado, 3 neutral, 4 muy afectado y 5 totalmente afectado. Los resultados se muestran en la figura número 5.

Tabla No. 2. Grado de afectación de las actividades extraescolares

\begin{tabular}{|c|c|}
\hline Nivel de afectación & Número de alumnos \\
\hline 1 & 104 \\
\hline 2 & 103 \\
\hline 3 & 194 \\
\hline 4 & 115 \\
\hline 5 & 132 \\
\hline Total & $\mathbf{6 4 8}$ \\
\hline
\end{tabular}


Figura 5. Grados de afectación de actividades extraescolares

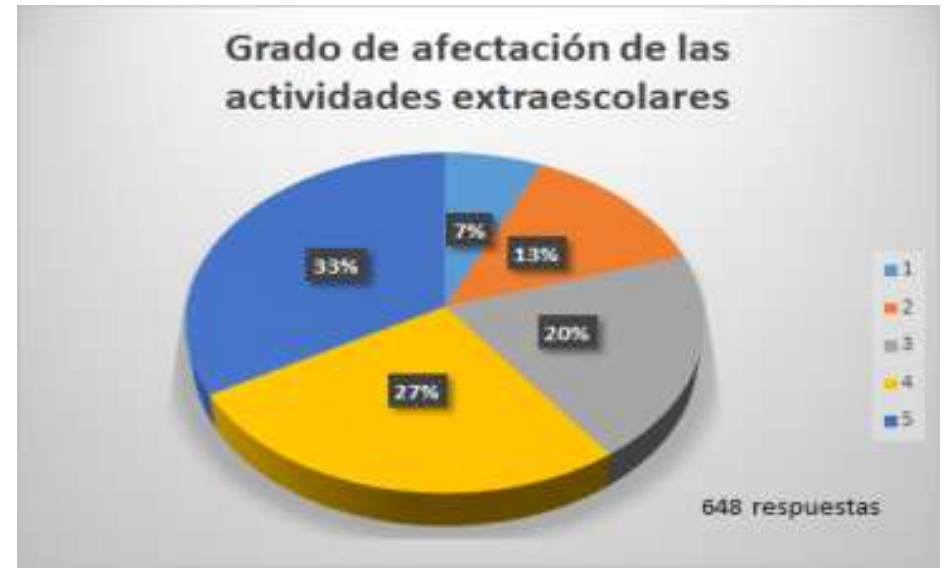

Fuente: Elaboración propia

La figura número 6 muestra las respuestas emitidas por los estudiantes referentes a las actividades extraescolares que han dejado de hacer a causa del confinamiento, 177 personas expresaron dejar de asistir al gimnasio, seguida esta cifra por 174 que dicen no haber dejado sus aficiones, 113 abandonaron los entrenamientos futbolísticos y 57 a competencias deportivas, dentro del rubro de la recreación artística, 67 no se ocuparon de sus clases de música o canto, 24 de clases de baile y solo 14 de clases de pintura. Los pasatiempos restantes no acumularon cifras significativas.

Figura 6. Qué dejaron de hacer los estudiantes

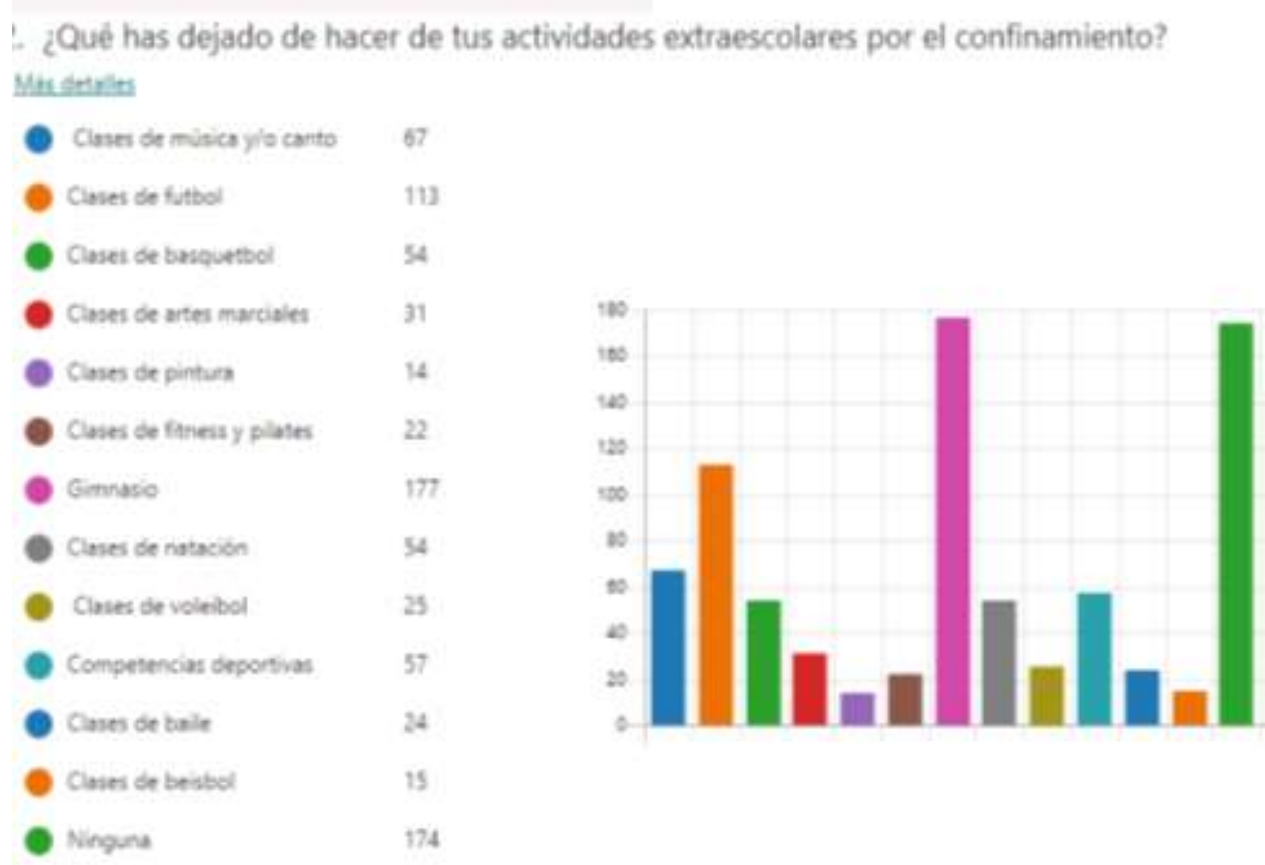

Fuente: Elaboración propia 
Los estudiantes hacen referencia que, lo que más extrañan hacer debido al confinamiento, es convivir con sus amistades (421), 374 jóvenes mencionan que dejaron de interactuar con otras personas, no se divirtieron ni salieron de paseo 342, mientras que 144 dicen que necesitaban abrazar a quién quieren. Sorprende que no fue relevante para ellos el visitar a la familia, ya que, de las 648 respuestas, únicamente se obtuvieron 79 decisiones en este ítem. 132 entregas fueron para el enunciado "No acompañar en momentos difíciles". A 41 personas no han extrañado nada y para 4 estudiantes fue relevante no hacer ejercicio. Figura 7.

Figura 7. Lo que extrañan hacer los estudiantes

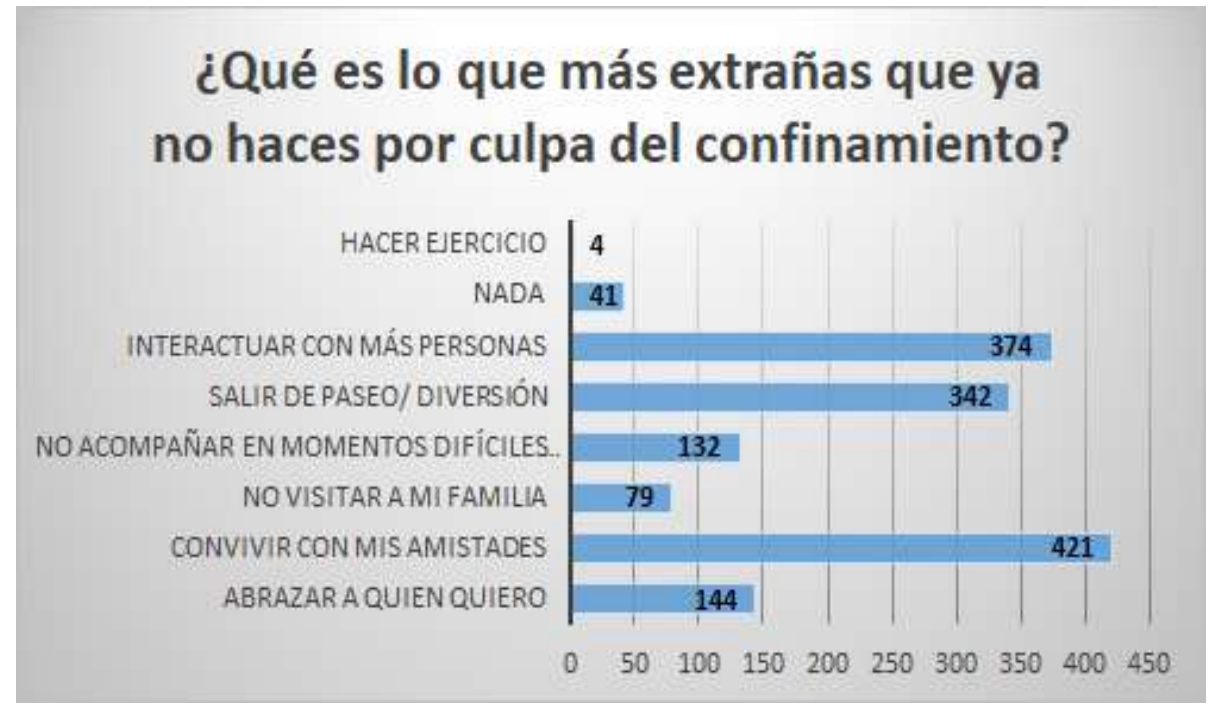

Fuente: Elaboración propia

La comunidad estudiantil reconoce no haber tenido afectaciones en el núcleo familiar (334) aunque, 96 dicen tener mala comunicación familiar y 143 expresan tener disgustos y conflictos por cambios de hábitos en horarios de sueño. 118 jóvenes que se negaron a realizar labores domésticas tuvieron enojos frecuentes por este motivo. Dos aspectos interesantes que revela la encuesta es que 112 estudiantes tienen poca tolerancia a estar en familia mientras que 105 pelean frecuentemente con padres y hermanos. A su vez, 90 dicen tener disgustos e incomodidad por falta de espacio físico, 65 expresan disgusto y conflictos por mala situación económica y solamente 20 se muestran molestos por falta de higiene personal en miembros de la familia. Esta información se muestra en la figura 8. 
Figura 8. Afectación de la relación familiar

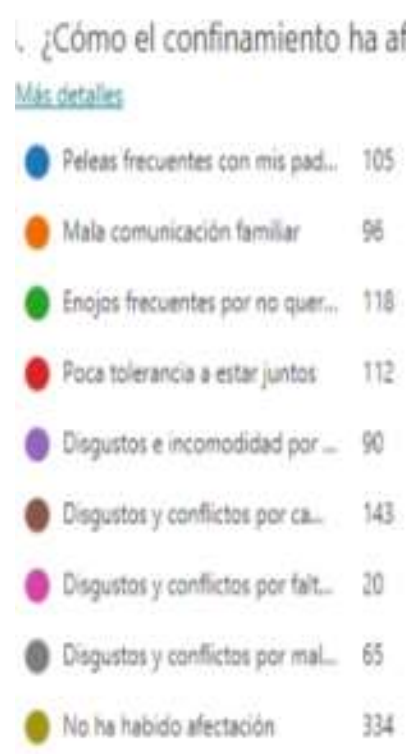

Fuente: Elaboración propia

Aunque pareciera ser desastroso que una familia numerosa se encontrara en casa por motivo de la pandemia, los estudiantes lograron observar beneficios, entre los que se encuentra el pasar tiempo con la familia, ítem que alcanzó 308 selecciones, seguida de experimentar nuevas habilidades como cocinar y tocar un instrumento (286). 210 personas se dedicaron a sus pasatiempos y 166 afirman que aumentaron sus horas de descanso, entre otros. Estos resultados se aprecian en la figura 9.

Figura 9. Beneficios de estar en casa 17 meses
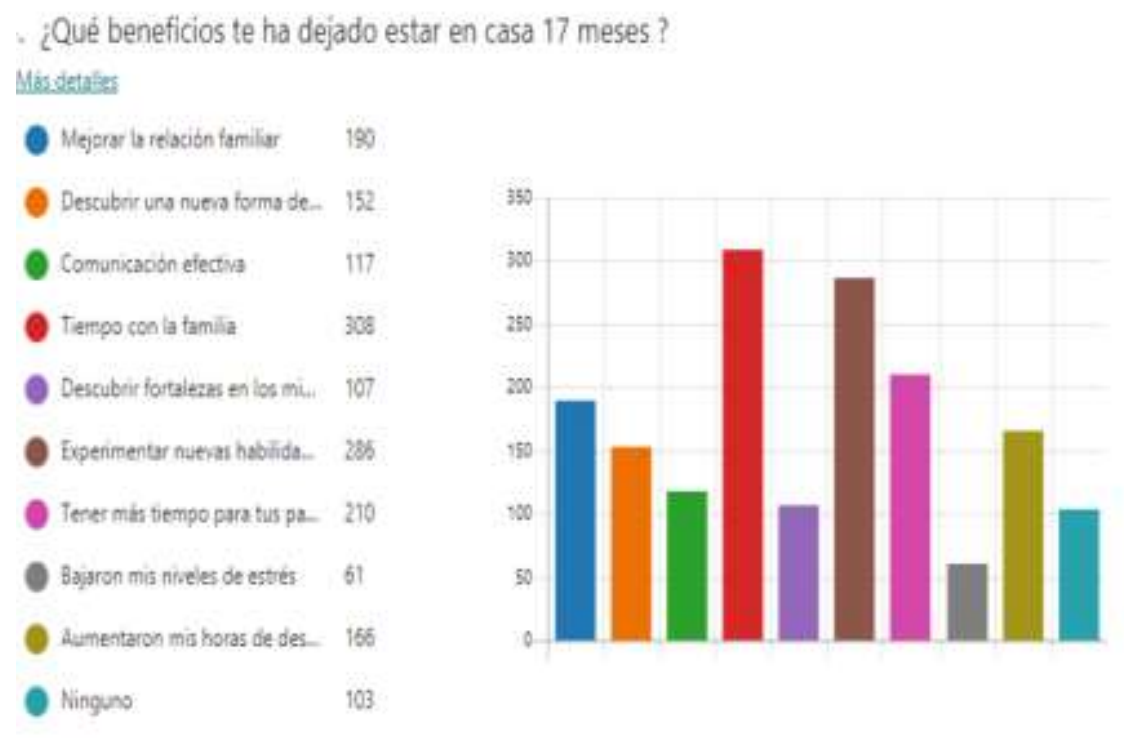

Fuente: Elaboración propia 
Para inicios del segundo semestre de 2021, los jóvenes aún no están vacunados en su totalidad con 2 dosis, solo 26 (4\%) las han recibido. La mayoría cuenta solo una dosis, $65 \%$ (422) y $200(31 \%)$ no están vacunados. Existe la posibilidad de que jóvenes con enfermedades autoinmunes, voluntarios en campañas a otros rangos de edades, embarazadas o asistentes médicos puedan ya tener el esquema completo de vacunación. Figura 10.

Figura 10. Dosis de vacuna recibidas

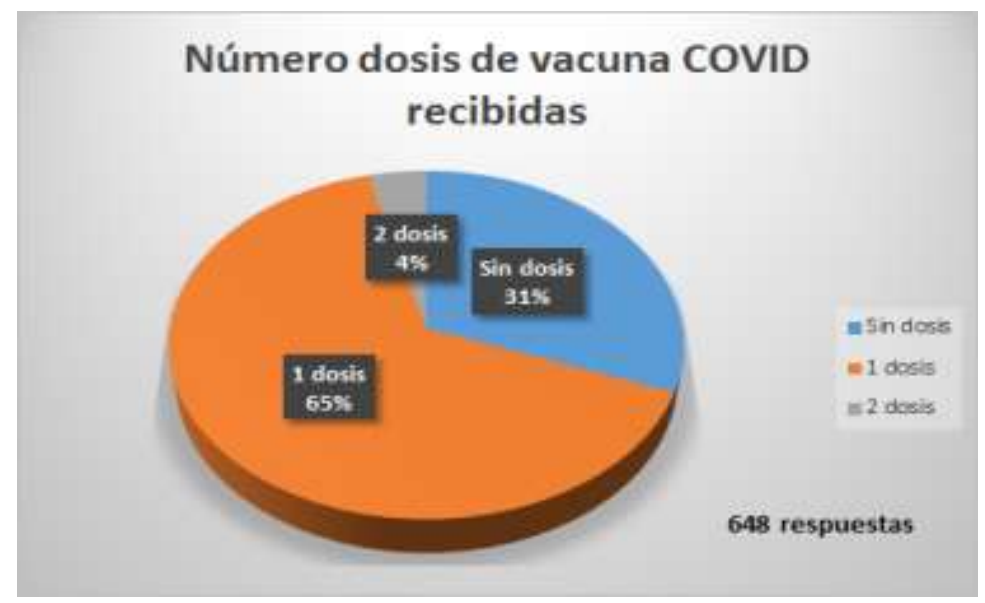

Fuente: Elaboración propia

La Secretaría de Educación Pública (SEP) ha planteado en repetidas ocasiones que el regreso a clases presenciales se realizará hasta que haya semáforo verde en las entidades. El gobierno de México se pronunció a favor del regreso a clases presenciales a partir del mes de agosto 2021 (Revista Expansión Política, 2021). Ante este escenario 413 (64\%) de los estudiantes les gustaría regresar a las aulas, mientras que 161 (25\%) se muestra indiferente y $74(11 \%)$ prefiere quedarse en casa. Figura 11.

Figura 11. Opinión sobre el regreso a clases presenciales

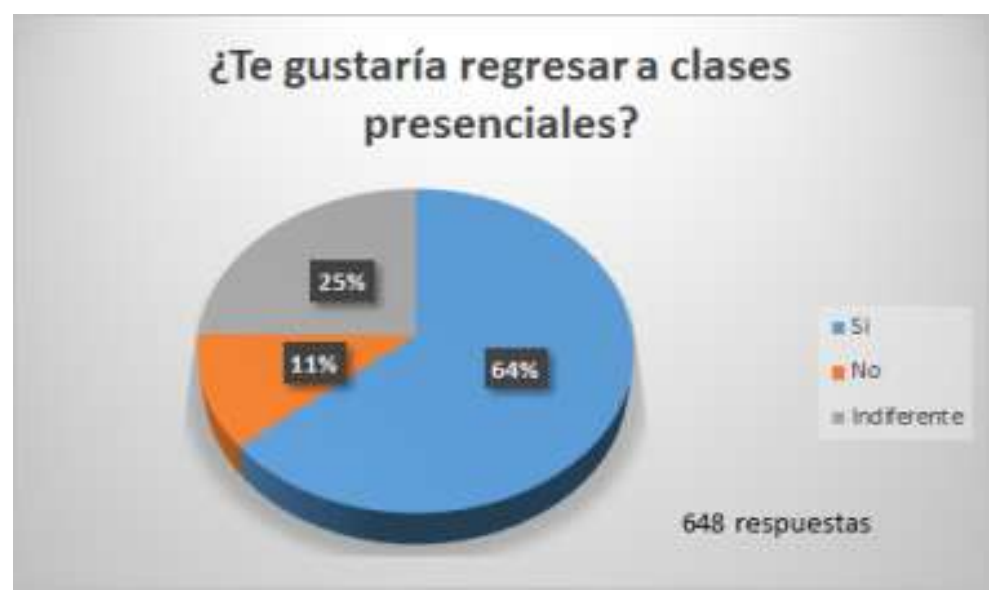

Fuente: Elaboración propia 
El 24 de mayo pasado, después del anuncio del regreso a clases por parte de la SEP, más de tres mil integrantes de la Federación Nacional de Estudiantes Revolucionarios "Rafael Ramírez" (FNERRR) marcharon en la Ciudad de México para exigir que se reconsidere la decisión de las autoridades educativas. La principal demanda de la organización es que se vacune a los estudiantes. Explican que, además de arriesgar sus vidas, los jóvenes podrían contagiar de COVID-19 a familiares no vacunados o con comorbilidades —obesidad o diabetes_- que agravarían la enfermedad (Bustamante, 2021).

Coincidiendo con estas opiniones, 278 de los encuestados están temerosos de contagiarse y contagiar a sus familiares, contrariamente 243 se encuentran entusiasmados por la reanudación de actividades escolares. La expectativa del retorno, 180 se manifiestan ansiosos y 169 inseguros ante el regreso a clase. Figura 12.

Figura 12. Cómo se sienten con la expectativa de regresar a clases presenciales

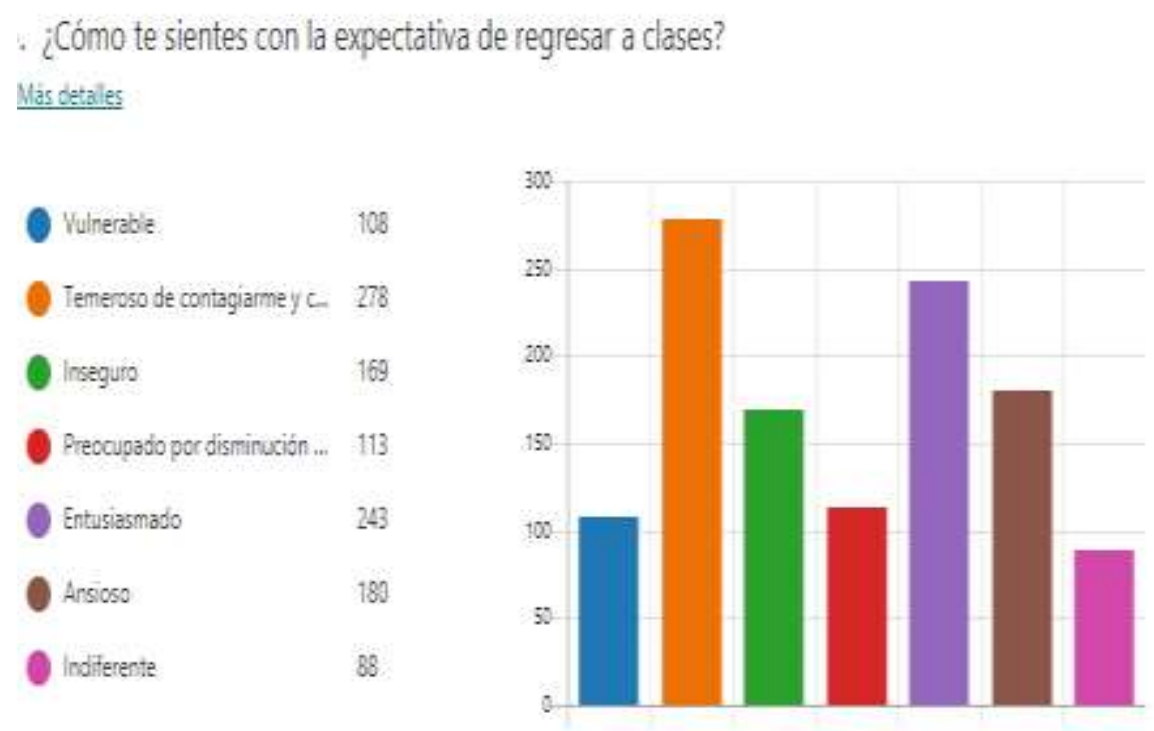

Fuente: Elaboración propia

\section{CONCLUSIÓN O CONSIDERACIONES FINALES}

Luego del estudio realizado queda claro que el confinamiento por la pandemia del COVID19 ha causado desequilibrio en la vida de los estudiantes, incidiendo principalmente en sus emociones. El trabajo demuestra manifestaciones de inestabilidad en fase inicial de salud mental de algunos jóvenes. Ante esto, las autoridades educativas y de salud deben ocuparse de planear el adecuado regreso a las aulas incluyendo capacitación a docentes, tutores y personal de psicopedagogía para que puedan brindar los primeros auxilios psicológicos y canalizar los casos que así lo requieran a las instancias correspondientes, buscando prevenir 
además la afectación del estado de salud físico y con esto disminuir el índice de ausentismo y deserción de las aulas.

En el sitio (UNICEF México, 2020), se sugieren los siguientes puntos como medidas precautorias para el cuidado de la salud mental:

- Organizar las labores y actividades del día, mantente ocupado en actividades productivas y positivas.

- Evitar el aburrimiento, ya que esté puede desatar pensamientos negativos y si te atacan, concéntrate, cuenta hasta diez y tranquilízate.

- Dedicar tiempo a pasatiempos que se pueden realizar en casa: leer, dibujar, bailar, oír música, ver vídeos, películas o series.

- Aceptar que cierta dosis de miedo y tristeza son saludables ya que son un instinto de supervivencia.

- Interactuar con las personas que vives y/o amigos, intentando ayudar, animar, apoyar. Comparte tiempo con la familia, colabora en las tareas del hogar, practica la tolerancia, solidaridad y respeto.

- Escribir un diario de emociones. Este te ayudará a expresar y desahogar tus sentimientos.

- Hay que recordar que todo pasa, nada es permanente.

- Evitar enterarse de noticias sobre fallecimientos e infectados, sobre todo cuando procede de fuentes no confiables.

Cada país ha desarrollado políticas de salud pública para atender a la población en general y México no es la excepción. Se aconseja en (Gobierno de México: Salud Mental, s.f.): "No lo olvides, una mente tranquila, es una mente saludable". En caso de requerir apoyo emocional se puede contactar las diversas líneas de atención que sugiere el sitio. En caso de presentar alguno de los síntomas de forma severa, indica (Paricio, 2020) se debe tener acceso a la valoración por parte de un profesional de la salud mental; empleando, siempre que resulte posible, la teleasistencia.

El regreso a las aulas debiera darse cuando se garantice la seguridad y salud para todos los involucrados. Educación 2020 y América Solidaria, con el apoyo de UNICEF, lideraron el proyecto "Recreando el Aprendizaje en Pandemia". Trabajaron durante tres meses con diferentes actores del área educativa para establecer un decálogo hacia el retorno seguro a clases presenciales. Las conclusiones de este equipo de trabajo empatan con algunas de las 
recomendaciones emitidas y son las siguientes:

- 5.- Entregar apoyo orientado al bienestar sicológico de la comunidad educativa y realizar campañas que prioricen el bienestar socioemocional.

- 10.- Monitorear el bienestar emocional de estudiantes y profesores en apoyo con la red de salud (UNICEF, 2021).

El compromiso de cuidar la salud es de todos. Estas recomendaciones son indispensables en su implementación y vigilancia para minimizar los riesgos de contagio. Considerando la etapa de adultez emergente de los encuestados, su grado de compromiso es mayor que el de los niveles de estudio precedentes, lo que representa una ventaja para garantizar el regreso seguro a la educación presencial.

\section{LISTA DE REFERENCIAS}

Apaza, C. S.-C. (2020). Factores psicosociales durante el confinamiento por el Covid-19Perú. Revista Venezolana de Gerencia, 25(90), 402-413. Recuperado el 17 de septiembre de 2021 , de https://www.redalyc.org/journal/290/29063559022/29063559022.pdf

Bustamante, A. A. (12 de junio de 2021). Universitarios: Entre el riesgo del COVID-19 y las ganas de volver a las aulas. Corriente Alterna. Recuperado el septiembte de 18 de 2021, de https://corrientealterna.unam.mx/derechos-humanos/regreso-a-clasesuniversidad-riesgo-covid19-jovenes/

Educación 2020. (29 de abril de 2020). Educación 2020. Recuperado el 16 de septiembre de 2021, de https://educacion2020.cl/noticias/encuesta-educacion-2020-y-estado-deanimo-de-estudiantes-ante-la-pandemia-63-se-siente-aburrido-y-solo-un-3-esta-felizen-casa/

Fernández, A. (2020). 2020: Estudiantes, emociones, salud mental y pandemia. Revista Andina de Educación, 4(1), 23-29. Recuperado el 16 de septiembre de 2021, de https://revistas.uasb.edu.ec/index.php/ree/article/view/1567/2302

Fundación Baltazar y Nicolás. Contribuyendo al desarrollo infantil temprano. (17 de diciembre de 2020). Fundación Baltazar y Nicolás. Recuperado el 17 de septiembre de 2021, de Importancia del contacto físico madre-hijo: https://misprimerostres.org/categoria/cuidados-bebe/contacto-fisico-madre-hijodurante-cuidado/

Gobierno de México: Salud Mental. (s.f.). Recuperado el 18 de septiembre de 2021, de 
https://coronavirus.gob.mx/salud-mental/

Hernández-Sampieri R., F. P. (2018). Metodología de la Investigación. Las ruas cuantitativas, cualitativa y mixta. (1a. ed.). México: Mc Graw Hill.

iisue. (2020). Educación y Pandemia: Una visión académica. (J. G. Palau, Ed.) Ciudad de México, Delegación Coyoacan, México: UNAM. Recuperado el 16 de septiembre de 2021, de https://www.iisue.unam.mx/investigacion/textos/educacion_pandemia.pdf

Jiménez, A. R. (11 de marzo de 2019). Problemas de salud mental en estudiantes universitarios(I): ¿consecuencias de la (sobre) carga académica? Recuperado el 15 de septiembre de 2021, de https://www.ciperchile.cl/2019/03/11/problemas-de-saludmental-en-estudiantes-universitarios-i-consecuencias-de-la-sobre-carga-academica/

Liberman, J. L.-B. (27 de abril de 2020). Education for Global Development. Recuperado el 17 de septiembre de 2021, de ¿Siguen aprendiendo los estudiantes durante el COVID-19? La respuesta podría obtenerse a través de la evaluación formativa: https://blogs.worldbank.org/es/education/siguen-aprendiendo-los-estudiantesdurante-el-covid-19-la-respuesta-podria-obtenerse

Mac-Ginty, S. J. (marzo de 2021). Impacto de la pandemia por COVID-19 en la salud mental de estudiantes universitarios en Chile. Revista chilena de Psiquatría y Neurología de la infancia y la adolescencia, 32(1), 23-37. Recuperado el 15 de septiembre de 2021, de https://www.sopnia.com/noticias/revistas/vol-no32n1/

Miguel, J. (4 de septiembre de 2020). La educación superior en tiempos de pandemia: una visión desde dentro del proceso formativo. Ibero. Revista Latinoamericana de $\begin{array}{llr}\text { Estudios } \quad \text { Educativos, } & \text { 50(ESPECIAL), }\end{array}$ doi:https://doi.org/10.48102/rlee.2020.50.ESPECIAL.95

OECD. (2020). oecd.org. Recuperado el 15 de septiembre de 2021, de El impacto del COVID-19 en la educación-Información del Panorama de la Educación (Education at a Glance): https://www.oecd.org/centrodemexico/medios/EAG2020_COVID\%20Brochure\%20 ES.pdf

OECD. (2021). OECDiLibrary. (OECDPublishing, Editor) doi:https://doi.org/10.1787/1a23bb23-en

Paricio, R. P. (30 de junio de 2020). Salud Mental infanto-juvenil y pandemia de Covid-19 en España: cuestiones y retos. (C. Editorial, Ed.) Revista de Psiquiatría Infanto- 
Juvenil, 37(2), 30-44. doi:http://dx.doi.org/10.31766/revpsij.v37n2a4

Pariente, E. (7 de junio de 2021). Depresión en universitarios durante la pandemia: "La adultez emergente es un periodo crítico en terminos vitales". (L. Tercera, Ed.) Recuperado el 15 de septiembre de 2021, de https://www.latercera.com/paula/depresion-en-universitarios-durante-la-pandemiala-adultez-emergente-es-un-periodo-critico-en-terminos-vitales/

Pediatrics, A. A. (Ed.). (8 de agosto de 2021). healtychildren.org. Recuperado el 19 de septiembre de 2021, de La salud mental durante el COVID-19: señales de que su adolescente pudiera necesitar más ayuda: https://www.healthychildren.org/Spanish/health-issues/conditions/COVID19/Paginas/Signs-your-Teen-May-Need-More-Support.aspx

Revista Expansión Política. (22 de febrero de 2021). Escuelas privadas anuncian regreso a clases el 1o. de marzo. Revista Expansión Política. Recuperado el 18 de septiembre de 2021, de https://politica.expansion.mx/mexico/2021/02/22/escuelas-privadasanuncian-regreso-a-clases-presenciales-el-1-de-marzo

Torrecilla, M. (25 de septiembre de 2020). Psicologo en Majadahonda. Recuperado el 17 de septiembre de 2021, de La importancia del contacto físico y el distanciamiento social: https://psicologoenmajadahonda.es/2020/09/25/la-importancia-del-contactofisico-y-el-distanciamiento-social/

UNESCO. (13 de mayo de 2020). COVID-19 y Educación Superior: de los efectos inmediatos al día después. Análisis de impacto, respuestas políticas y recomendaciones. Recuperado el 16 de septiembre de 2021, de https://www.iesalc.unesco.org/wp-content/uploads/2020/05/COVID-19-ES130520.pdf

UNICEF. (28 de enero de 2021). UNICEF. (UNICEF, Editor) Recuperado el 18 de septiembre de 2021, de 10 Recomendaciones para el retorno a clases en 2021: https://www.unicef.org/chile/comunicados-prensa/10-recomendaciones-para-elretorno-clases-en-2021

UNICEF México. (1 de abril de 2020). Salud mental y emocional de las y los adolescentes ante el COVID-19 [Vídeo YouTube]. Recuperado el 16 de septiembre de 2021, de https://www.youtube.com/watch?v=EJ48mIG8ksA 